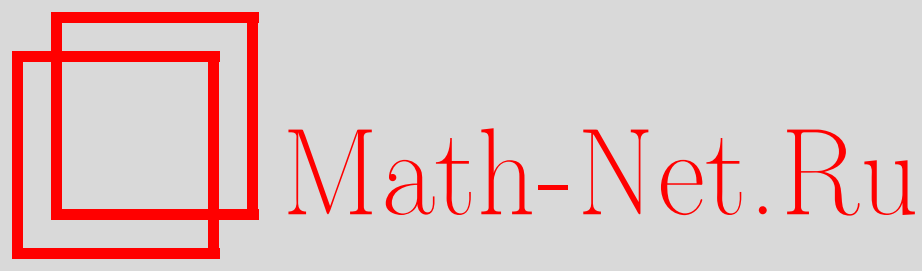

В. М. Бухштабер, С. И. Тертычный, Семейство явных решений уравнения резистивной модели перехода Джозефсона, ТМФ, 2013, том 176, номер 2, 163-188

DOI: https://doi.org/10.4213/tmf8512

Использование Общероссийского математического портала Math-Net.Ru подразумевает, что вы прочитали и согласны с пользовательским соглашением http: //www . mathnet.ru/rus/agreement

Параметры загрузки:

IP: 18.234 .197 .8

26 апреля 2023 г., 06:28:18

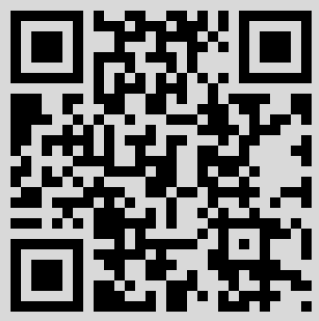




\title{
СЕМЕЙСТВО ЯВНЫХ РЕШЕНИЙ УРАВНЕНИЯ РЕЗИСТИВНОЙ МОДЕЛИ ПЕРЕХОДА ДЖОЗЕФСОНА
}

\begin{abstract}
Получено и исследовано семейство решений уравнения $\dot{\phi}+\sin \phi=B+A \cos \omega t$, которое применяется в ряде задач физики, механики и геометрии. Это семейство строится по полиномиальным решениям дважды конфлюентного уравнения Гойна, ассоциированного с данным уравнением. Описано многообразие $M_{\mathrm{P}}$ параметров $(A, B, \omega)$ таких решений. Получены явные формулы для числа вращения и для отображения Пуанкаре динамической системы на торе, соответствующей указанному уравнению при $(A, B, \omega) \in M_{\mathrm{P}}$.
\end{abstract}

Ключевые слова: динамическая система на торе, дважды конфлюентные уравнения Гойна, полиномиальные решения, число вращения, отображение Пуанкаре.

DOI: $1010.4231 / \operatorname{tmf} 8512$

\section{1. ВВЕДЕНИЕ}

Работа посвящена построению и исследованию свойств семейства явных решений дифференциального уравнения

$$
\dot{\phi}(t)+\sin \phi(t)=B+A \cos \omega t,
$$

где $A, B$ и $\omega$ - вещественные константы. Используя группу симметрии уравнения (1) [1], нетрудно показать, что достаточно исследовать это уравнение только при рассмотренных ниже условиях, а именно при $A \geqslant 0, B \leqslant 0, \omega>0$. Данное уравнение и его обобщения вида $\dot{\phi}(t)+\sin \phi(t)=f(t)$, где $f(t)$ - некоторая заданная функция, встречаются в ряде задач физики, механики и геометрии. Наиболее известным является применение уравнения (1) в составе так называемой резистивной модели перехода Джозефсона [2]-[6] в случаях, когда влиянием емкости перехода можно пренебречь (так называемый сильношунтированный переход). Другим примером является исследование геометрическими методами механики некоторых систем с неголономной связью. Это модель так называемого планиметра Притца [7],

* Математический институт им. В. А. Стеклова РАН, Москва, Россия. E-mail: buchstab@mi.ras.ru

†ФГУП "Всероссийский научно-исследовательский институт физико-технических и радиотехнических измерений”, Менделеево, Московская обл., Россия 
упрощенная модель кинематики движения велосипеда [8]. Аналогичным уравнением описывается наблюдаемое направление на точку в бесконечности при движении вдоль прямой в плоскости Лобачевского [7].

Сравнительно недавно была установлена особая роль уравнения (1) как уравнения интегральных кривых соответствующего векторного поля на двумерном торе. Положив $x=\omega t+c$, где $c$ - вещественная константа, и $y=\phi(t)$, мы получаем, что при $\omega A \neq 0$ уравнение (1) эквивалентно динамической системе на плоскости $\mathbb{R}^{2}$

$$
\dot{x}=\omega, \quad \dot{y}=B+A \cos x-\sin y,
$$

задающей векторное поле без особых точек на двумерном торе $\mathbb{T}^{2}$. Система (2) исследовалась в работе [9] с точки зрения теории быстро-медленных систем. Было обнаружено, что при малых значениях $\omega$ в потоке векторного поля этой системы формируются так называемые "циклы-утки".

Динамическая система на торе задает диффеоморфизм окружности $H: S^{1} \rightarrow S^{1}$ (отображение Пуанкаре), и для нее определено число вращения

$$
N=\lim _{t \rightarrow \infty} \frac{y(t)}{x(t)},
$$

где $(x(t), y(t))$ - решение соответствующей системы на плоскости. Число вращения не зависит от начальных данных $(x(0), y(0))$ и является непрерывной функцией от диффеоморфизма $H$ [10], [11]. Таким образом, в случае системы (2) мы имеем непрерывную функцию $N=N(A, B, \omega)$ в $\mathbb{R}^{3}$. Известно, что $N(0, B, \omega)=0$, если $|B| \leqslant 1$ и $N(0, B, \omega)=\operatorname{sgn} B \sqrt{B^{2}-1}$ при $|B|>1$. Свойства этой функции, вытекающие из вида системы (2), описаны в работе [1].

Число вращения $N$ является рациональным тогда и только тогда, когда диффеоморфизм $H^{m}$ для некоторого натурального $m$ имеет неподвижную точку, т. е. когда система имеет на торе периодическое решение. В общем случае система на торе является структурно устойчивой тогда и только тогда, когда ее число вращения рационально и все периодические решения невырожденны [11]. Как оказалось, в отличие от динамических систем общего положения, для системы (2) условие того, что при данных значениях параметров $(A, B, \omega)$ число вращения $N(A, B, \omega)$ целое, является необходимым условием структурной устойчивости [12], [13]. В резистивной модели перехода Джозефсона число $N$ получает физическую интерпретацию как номер так называемой ступеньки Шапиро. Таким образом, замыкание областей структурной устойчивости (резонансного захвата) для системы (2) задается уравнениями $N(A, B, \omega)=m, m=0, \pm 1, \ldots$. Указанное свойство системы (2) было названо в работе [12] эффектом квантования числа вращения. Как показано в работах [13], [14], система

$$
\dot{x}=\omega, \quad \dot{y}=B+A \cos x-\sin y-\alpha \sin 2 y
$$

этим свойством не обладает для сколь угодно малого $\alpha$. В то же время эффектом квантования обладают числа вращения системы

$$
\dot{x}=\omega, \quad \dot{y}=q(x)-\sin y,
$$

где $q(x)$ - любая достаточно регулярная $2 \pi$-периодическая функция. Доказательство этого факта в работе [12] опирается на следующий общий результат [15]. Пусть 
$q(t)$ - некоторая вещественная функция. Функции $\phi(t)$ и $\xi(t)$ удовлетворяют системе

$$
\dot{\phi}(t)+\sin \phi(t)+q(t)=0, \quad \dot{\xi}(t)-\cos \phi(t)=0
$$

с начальными условиями $\phi(0)=\phi_{0}, \xi(0)=1$ тогда и только тогда, когда функции $u(t)=\rho(t) \cos \psi(t)$ и $v(t)=\rho(t) \sin \psi(t)$, где $\rho(t)=e^{\xi(t) / 2}$ и $\psi(t)=\left(\phi(t)-\phi_{0}\right) / 2$, являются решением линейной системы

$$
2 \dot{u}=a u+(q-b) v, \quad-2 \dot{v}=(q+b) u+a v
$$

с начальными условиями $u(0)=1, v(0)=0$, где $a=\cos \phi_{0}, b=\sin \phi_{0}$ - константы.

В работе [16] было впервые указано на связь уравнения (1) с семейством дважды конфлюентных уравнений Гойна - линейных дифференциальных уравнений второго порядка [17], [18]; необходимое обоснование приведено в работе [19]. Теория уравнений Гойна (K. L.W. Heun), в том числе дважды конфлюентных уравнений, с описанием физических моделей, приводящих к этим семействам, обсуждается в монографии [18]. Результаты работы [20] позволяют получить общее решение уравнения (1) в случае резонансного захвата фазы.

В настоящей работе построены полиномиальные решения дважды конфлюентного уравнения Гойна и соответствующие им решения уравнения (1), которые, повидимому, являются новыми, за исключением очевидного решения $\phi=\pi / 2-\omega t$ (случай $A=1, B=-\omega)$. Описано подмногообразие $M_{\mathrm{P}} \subset \mathbb{R}^{3}$ параметров $(A, B, \omega)$, при которых полиномиальные решения существуют. Показано, что подмногообразие $M_{\mathrm{P}}$ принадлежит границе, разделяющей области резонансного захвата и псевдохаотического поведения решений уравнения (1). Приведены явные формулы для числа вращения и отображения Пуанкаре динамической системы $(2)$ при $(A, B, \omega) \in M_{\mathrm{P}}$. Отображения Пуанкаре представлены в терминах модифицированных функций Бесселя первого рода. В приложении А приведены демонстрационные примеры, в приложении Б описаны свойства решений уравнения (1) в терминах системы (3), в приложении В рассмотрен предельный случай $A \rightarrow 0$.

\section{2. МЕТОД ПОСТРОЕНИЯ И ИССЛЕДОВАНИЯ РЕШЕНИЙ}

В основе использованного в нашей работе метода лежит следующий результат.

ЛЕмма 1. Пусть $P(z)$ есть аналитическая вещественная (т.е. удовлетворяющая условию $\overline{P(z)}=P(\bar{z}))$ функиия, определенная в открытой окрестности единичной окружности $S^{1}=\{z \in \mathbb{C}:|z|=1\}$ на комплексной плоскости $\mathbb{C}$, такая, что

$$
P^{\prime}(z)=\mu P(z)+(2 \omega)^{-1} \epsilon z^{n} P\left(z^{-1}\right),
$$

где $\mu \neq 0$ и $\omega>0$ - вещественные константы, $n$ - целое число, $a \epsilon^{2}=1$. Тогда справедливы следующие утверждения.

1. Функиия $P(z)$ имеет аналитическое продолэение на всю комплексную плоскость, кроме, возможно, ее центра.

2. $П р u$

$$
A=2 \omega \mu, \quad B=-(n+1) \omega
$$

функция

$$
\Phi(z)=i \epsilon z^{-(n-1)} \frac{P(z)}{P\left(z^{-1}\right)}
$$


задает вещественно-аналитическое решение $\phi(t)$ уравнения (1), которое при всех $t$ удовлетворяет уравнению

$$
e^{i \phi(t)}=\Phi\left(e^{i \omega t}\right)
$$

$u$, следовательно, на некотором интервале изменения $t \geqslant 0$ выражается формулой $\phi(t)=\arg \Phi\left(e^{i \omega t}\right)$, причем

$$
\arg \Phi\left(e^{i \omega t}\right) \equiv-\frac{1}{2} \epsilon \pi-(n+1) \omega t+2 \arg P\left(e^{i \omega t}\right) \quad \bmod 2 \pi .
$$

В лемме 1 и далее штрих обозначает производную аналитической функции по ее аргументу.

ЗАмечАниЕ 1 . Требование, что $n$ - целое число, по существу является не частью условий леммы, а ее следствием. Действительно, если $n$ не целое, но остальные условия соблюдены, выражение в правой части равенства (4) есть многозначная функция, меняющая свое значение, когда $z$ обходит единичную окружность, в то время как выражение в левой части есть однозначная функция.

По ходу доказательства и в дальнейшем нам понадобится еще одна лемма, которую мы докажем в первую очередь.

ЛЕмма 2. Функция $P(z)$, удовлетворяющая условиям леммы 1 , не имеет нулей на единичной окружности.

ДокАЗАТЕЛьство. Пусть существует корень $z_{0}$ кратности $m \geqslant 1$ функции $P(t)$, где $\left|z_{0}\right|=1$. Тогда $z_{0}$ есть корень кратности $m-1$ производной $P^{\prime}(z)$ при $m>1$ и $P^{\prime}\left(z_{0}\right) \neq 0$ при $m=1$. Из уравнения (4) вытекает равенство

$$
P\left(z^{-1}\right)=2 \omega \epsilon z^{-n}\left(P^{\prime}(z)-\mu P(z)\right),
$$

из которого очевидным образом следует, что $z_{0}$ есть корень кратности $m-1$ функции $\widetilde{P}(z)=P\left(z^{-1}\right)$ при $m>1$, а при $m=1$ значение $z_{0}$ корнем данной функции не является. Эквивалентно, $z_{0}^{-1}$ есть корень кратности $m-1$ функции $P(z)=\widetilde{P}\left(z^{-1}\right)$ при $m>1$ и не является корнем данной функции при $m=1$. Но $\left|z_{0}\right|=1$, поэтому $z_{0}^{-1}=\bar{z}_{0}$. Из уравнения (4) получаем теперь, что $\bar{z}_{0}$ является корнем кратности $m-1$ функции $P(z)$ при $m>1$ и не является ее корнем при $m=1$. Если значение $z_{0}$ вещественно, то это напрямую противоречит исходному предположению. Пусть теперь значение $z_{0}$ комплексно, тогда, поскольку корни аналитической вещественной функции существуют в парах со своими комплексно-сопряженными и кратности элементов этих пар одинаковы, мы снова приходим к противоречию. Таким образом, функция $P(z)$ не имеет корней, по модулю равных единице. Лемма 2 доказана.

ДоказАтельство ЛЕммы 1. Наряду с уравнением (4) в некоторой окрестности единичной окружности выполняется также эквивалентное ему соотношение

$$
P^{\prime}\left(z^{-1}\right)=\mu P\left(z^{-1}\right)+(2 \omega)^{-1} \epsilon z^{-n} P(z)
$$

которое получается путем подстановки $z \rightarrow z^{-1}$. Продифференцируем уравнение (4) по $z$ и в получившемся выражении заменим с помощью (4) производную $P^{\prime}\left(z^{-1}\right)$ на линейную комбинацию функций $P(z)$ и $P\left(z^{-1}\right)$, где затем заменим $P\left(z^{-1}\right)$ на 
линейную комбинацию функций $P(z)$ и $P^{\prime}(z)$ с помощью уравнения (8). В итоге легко убедиться, что из уравнения (4) вытекает справедливость уравнения

$$
z^{2} P^{\prime \prime}(z)-\left(n z+\mu\left(z^{2}-1\right)\right) P^{\prime}(z)+(\mu n z+\lambda) P(z)=0, \quad \lambda=(2 \omega)^{-2}-\mu^{2} .
$$

Из него получаем следующее уравнение для функции $y(z)=z^{-(n+1) / 2} e^{-\mu z} P(z)$ :

$$
\left.D^{2} y+\alpha\left(z+z^{-1}\right) D y+\left(\left(\beta_{1}+\frac{1}{2}\right) \alpha z+\left(\frac{\alpha^{2}}{2}-\gamma\right)+\left(\beta_{-1}-\frac{1}{2}\right) \alpha z^{-1}\right)\right) y=0
$$

где

$$
D=z \frac{d}{d z}, \quad \alpha=\mu, \quad \beta_{1}=\frac{1}{2} n, \quad \beta_{-1}=\frac{1}{2} n+1, \quad \gamma=-\lambda-\frac{1}{2} \mu^{2}+\frac{1}{4}(n+1)^{2} .
$$

Если рассматривать $\alpha, \beta_{1}, \beta_{-1}$ и $\gamma$ как произвольные постоянные параметры, то уравнение (11) есть каноническое представление дважды конфлюентного уравнения Гойна (см. [17], уравнение (0.0.1)). Если положить $v=e^{-\mu z} P(z)$, то после дробнолинейного преобразования

$$
\zeta=\frac{z+1}{z-1}
$$

независимой переменной получается следующее уравнение, впервые приведенное в работе [16]:

$$
\left[\left(1-\zeta^{2}\right) \frac{d}{d \zeta}\left(1-\zeta^{2}\right) \frac{d}{d \zeta}-2\left((n+1)\left(1-\zeta^{2}\right)+2 \mu\left(1+\zeta^{2}\right)\right) \frac{d}{d \zeta}+\omega^{-2}\right] v=0 .
$$

Для уравнения (10) $z=0$ есть единственная сингулярная точка на комплексной плоскости. Если продолжить его на сферу Римана, то добавляется вторая сингулярная точка в бесконечности, “симметричная" первой относительно инверсии. Из (10) это сразу не следует, но данный факт вытекает из того, что точка $z=\infty$ является образом сингулярной точки $\zeta=1$ уравнения (13) при обратном к (12) преобразовании $\zeta \rightarrow z$. Вторая сингулярная для уравнения (13) точка $\zeta=-1$ соответствует $z=0$. Поскольку других сингулярных точек у уравнения (10) нет, точка $\zeta=\infty$ для уравнения (13) регулярна.

Таким образом, аналитическая функция, удовлетворяющая уравнению (4), удовлетворяет также уравнению (10), регулярному всюду, кроме центра комплексной плоскости (и бесконечности, если рассматривать сферу Римана). Интегрируя его ограничения на радиальные лучи и используя значения $P(z)$ и $P^{\prime}(z)$ на единичной окружности как начальные данные, можно продолжить область определения функции $P(z)$ на всю комплексную плоскость, кроме ее центра. По стандартной теореме о зависимости решений линейных обыкновенных дифференциальных уравнений от начальных данных полученное продолжение будет гладкой функцией переменных $\operatorname{Re} z$ и $\operatorname{Im} z$. Используя тот факт, что эта функция удовлетворяет уравнениям Коши-Римана в окрестности единичной окружности, можно показать, что она удовлетворяет этим уравнениям всюду, кроме, возможно, точки $z=0$. Следовательно, по крайней мере при $z \neq 0$, эта функция будет голоморфной. На единичной окружности она совпадает с аналитической функцией $P(z)$, следовательно, совпадение имеет место везде, где последняя функция была задана, и в результате мы 
получаем продолжение функции $P(z)$ на всю комплексную плоскость с выколотым центром.

Наконец, поскольку аналитическая функция $P^{\prime}(z)-\mu P(z)-(2 \omega)^{-1} \epsilon z^{n} P\left(z^{-1}\right)$ равна нулю в окрестности единичной окружности, она равна нулю тождественно, в том числе для полученного продолжения функции $P(z)$, и уравнение (4) выполняется всюду.

Обратимся теперь к построению решения уравнения (1) по формулам (6), (7). Как следствие лемм 1 и 2, выражение в правой части корректно определено и представляет собой аналитическую функцию переменной $z$ на единичной окружности (а также, по крайней мере, в некоторой ее окрестности). Ввиду вещественности функции $P(z)$ модуль этого выражения на единичной окружности равен 1. Далее, с помощью непосредственной проверки можно убедиться, что, как следствие соотношений (4) и (9),

$$
\omega z \Phi^{-1} \Phi^{\prime}=-(2 i)^{-1}\left(\Phi-\Phi^{-1}\right)-\omega\left(n+1-\mu\left(z+z^{-1}\right)\right) .
$$

Выражение в правой части последнего равенства есть явно заданная (выражаемая через функцию $P$ ) аналитическая, вещественнозначная и ограниченная на единичной окружности функция.

Пусть вещественное число $\phi_{0}$ удовлетворяет уравнению $e^{i \phi_{0}}=-i \epsilon$. Одно из допустимых значений есть $\phi_{0}=-\epsilon \pi / 2$, остальные получаются с помощью сдвигов на $2 \pi$. Рассмотрим функцию $\phi(t)$, определенную при любом $t \in \mathbb{R}$ квадратурой

$$
\phi(t)=\phi_{0}+\left.\int_{0}^{t}\left[-(2 i)^{-1}\left(\Phi-\Phi^{-1}\right)-\omega\left(n+1-\mu\left(z+z^{-1}\right)\right)\right]\right|_{z=e^{i \omega t}} d t
$$

где под $\Phi$ подразумевается выражение в правой части равенства (6). После такой подстановки подынтегральное выражение становится полностью определенной функцией от $z$. Интеграл от него вычисляется при $z=e^{i \omega t}$. Как следствие леммы 2 , знаменатели слагаемых, равные $P\left(e^{i \omega t}\right)$, нигде не обращаются в нуль, и для любого $t$ функция $\phi(t)$ корректно определена как вещественно-аналитическая функция. Из данного определения и формулы (14), в частности, следуют равенства

$$
\frac{d}{d t} \frac{e^{i \phi(t)}}{\Phi\left(e^{i \omega t}\right)}=0, \quad \frac{e^{i \phi(0)}}{\Phi(1)}=i \epsilon e^{i \phi_{0}} .
$$

Таким образом, $\Phi\left(e^{i \omega t}\right)=-i \epsilon e^{i \phi(t)}$ и, соответственно, $\phi(t)=\phi_{0}+\epsilon \pi / 2+i^{-1} \ln \Phi\left(e^{i \omega t}\right)$ при определенном выборе $\phi_{0}$ в пределах допустимых значений и до тех пор, пока аргумент логарифма остается внутри области его однозначной определенности, т. е. пока $\left|\arg \Phi\left(e^{i \omega t}\right)\right|<\pi$. Остается заметить, что при таком отождествлении ограничение уравнения (14) на единичную окружность представляет собой не что иное, как уравнение (1). Лемма 1 доказана.

СлеДСТВИЕ 1. Любая функиия $P(z)$, кроме тождественно равной нулю, удовлетворяющая условиям леммы 1, не имеет ненулевых кратных нулей.

Действительно, если $z_{0}$ - кратный ноль, то одновременно $P\left(z_{0}\right)=0$ и $P^{\prime}\left(z_{0}\right)=0$, и тогда при $z_{0} \neq 0$ из уравнения (10) следует, что $P(z) \equiv 0$. 
ЗАмЕчание 2. Уравнение (10) играет в рассматриваемой задаче более важную роль, чем просто следствие уравнения (4). Любое решение $P(z)$ дважды конфлюентного уравнения Гойна (10), кроме тривиального, независимо от того, удовлетворяет ли оно уравнению (4), можно связать с некоторым решением $\phi(t)$ уравнения (1) и наоборот. Соответствующее обобщение формулы (6) имеет вид

$$
\Phi(z)=z^{-1} \frac{2 \omega z^{n}\left(P^{\prime}\left(z^{-1}\right)-\mu P\left(z^{-1}\right)\right)-i P(z)}{2 \omega\left(P^{\prime}(z)-\mu P(z)\right)+i z^{n} P\left(z^{-1}\right)} .
$$

Легко видеть, что на единичной окружности решение $\phi(t)=i^{-1} \ln \Phi\left(e^{i \omega t}\right)$ уравнения (1) (корректно определенное, пока $t$ меняется в окрестности нуля) вещественно, если $P$ - вещественная аналитическая функция. Если функция $P$ последнему условию не удовлетворяет, то вместо нее в формуле (16) можно использовать, в частности, функцию $(P(z)+\overline{P(\bar{z})}) / 2$ - аналитическое продолжение с единичной окружности вещественной части функции $P(z)$. Поскольку коэффициенты линейного уравнения (10) вещественны, она будет его решением, как и $P(z)$. Параметры $(A, B, \omega)$ уравнения (1) выражаются через параметры $(n, \mu, \lambda)$ уравнения $(10)$ с помощью тех же формул (5) и второго равенства в (10), но вещественное число $n$ уже не обязано быть целым.

\section{3. ПОЛИНОМИАЛЬНЫЕ РЕШЕНИЯ}

В этом разделе мы построим и исследуем решения уравнения (4) в предположении, что $P(z)$ - полином с вещественными коэффициентами. Показать, что такие решения существуют, а заодно и определить, какова должна быть степень такого полинома, проще всего с помощью следующей эквивалентной записи уравнения (10):

$$
z\left(z P^{\prime}-n P\right)^{\prime}-\mu z\left(z P^{\prime}-n P\right)+(\mu-z) P^{\prime}+\lambda P=0 .
$$

Здесь дифференциальный оператор в левой части уравнения, действуя на полином, увеличивает его степень на единицу (некомпенсируемый прирост степени дает второе слагаемое), и равенство нулю получиться не может во всех случаях, за исключением одного, а именно, когда $n$ есть неотрицательное целое число и полином $P=P_{n}=P_{n}(z)$ имеет степень $n$ (или, по крайней мере, не выше $n$ ). В этом случае степень не увеличивается, и есть шанс подобрать коэффициенты в слагаемых полинома так, чтобы уравнение (17) удовлетворялось. Простейший пример случай $n=0$, для которого $P_{0}=$ const. Решение можно получить, но при ограничении $\lambda=0$ или, что эквивалентно, при $|2 \omega \mu|=1$. Мы имеем как раз случай упоминавшегося выше очевидного решения $\phi=\pi / 2-\omega t$. Для $n=0$ это (при $\mu>0$ ) единственная возможность.

Для любого натурального $n$ положим $P_{n}=\sum_{k=0}^{n} a_{k} z^{k}$, где $a_{k}$ - вещественные константы, подлежащие определению. Уравнение (17) превращается в следующую замкнутую систему $n+1$ однородных линейных уравнений относительно коэффициентов $a_{k}, k=0,1, \ldots, n$ :

$$
\begin{aligned}
& \lambda a_{0}+\mu a_{1}=0, \\
& \mu(n-k+1) a_{k-1}+(\lambda-k(n-k+1)) a_{k}+\mu(k+1) a_{k+1}=0, \quad k=1, \ldots, n-1, \\
& \mu a_{n-1}+(\lambda-n) a_{n}=0
\end{aligned}
$$


(при $n=1$ подсистема (19) пуста). Необходимым и достаточным условием существования нетривиального решения этой системы является обращение в нуль детерминанта $\Delta_{n}(n+1) \times(n+1)$-матрицы $\mathbb{H}_{n}+\lambda \mathbb{I}$, где $\mathbb{I}-$ единичная матрица, а $\mathbb{H}_{n}=\mathbb{H}_{n}(\mu)=\left(h_{k, l}\right)$, где

$$
h_{k, l}=\left\{\begin{array}{lll}
0, & \text { если }|k-l|>1, \\
-k(n-k+1), & \text { если } l=k, & k=0,1, \ldots, n, \\
\mu(l-1), & \text { если } k=l-1, & l=2,3, \ldots, n+1, \\
\mu(n-k+2), & \text { если } l=k-1, & k=2,3, \ldots, n+1 .
\end{array}\right.
$$

Это условие представляет собой алгебраическое уравнение степени $n+1$ по $\lambda$ при заданном $\mu$. Далее для удобства мы будем называть уравнение $\Delta_{n}(\lambda, \mu)=0$ cneктральным. При заданном $\mu$ существование вещественного корня $\lambda$ этого уравнения является необходимым и достаточным условием существования полиномиального решения уравнения (17) (или уравнения (10)) Очевидно, оно необходимо для построения решения уравнения (1) с помощью формул (6) и (7). Ниже будет доказана и его достаточность (см. следствие 4). Таким образом, искомые решения уравнения (1) соответствуют точкам $(\lambda, \mu) \in \mathbb{R}^{2}$ плоской алгебраической кривой в $\mathbb{C}^{2}$ с координатами $\lambda, \mu$. Первые несколько полиномов

$$
\Delta_{n}=\Delta_{n}(\lambda, \mu)=\operatorname{det}\left(\mathbb{H}_{n}(\mu)+\lambda \mathbb{I}\right)
$$

имеют вид

$$
\begin{aligned}
\Delta_{1} & =\lambda^{2}-\lambda-\mu^{2}=\lambda^{2}-(2 \omega)^{-2} \\
\Delta_{2} & =\lambda^{3}-4 \lambda^{2}-4 \lambda \mu^{2}+4 \lambda+4 \mu^{2}=\lambda^{3}-\omega^{-2} \lambda+\omega^{-2}, \\
\Delta_{3} & =\lambda^{4}-10 \lambda^{3}-10 \lambda^{2} \mu^{2}+33 \lambda^{2}+42 \lambda \mu^{2}-36 \lambda+9 \mu^{4}-36 \mu^{2}= \\
& =\lambda^{4}-\frac{5}{2} \omega^{-2} \lambda^{2}+6 \omega^{-2} \lambda-9 \omega^{-2}+\frac{9}{16} \omega^{-4},
\end{aligned}
$$

где параметры $\lambda, \mu$ и $\omega$ связаны соотношением

$$
4 \omega^{2}\left(\lambda+\mu^{2}\right)=1 \text {. }
$$

В дополнение к формулам (22) можно положить $\Delta_{0}=\lambda$. Из свойств матрицы $\mathbb{H}_{n}(\mu)$ (см. ниже) следует, что $\lambda+\mu^{2}>0$ при $\mu \neq 0$. Таким образом, уравнение (23) задает значение $\omega>0$.

Соответствующие полиномиальные решения уравнения (10) с параметрами $\mu$ и $\lambda$, где $\lambda$ - корень спектрального уравнения, имеют вид

$$
\begin{aligned}
P_{1}= & \mu z-\lambda+1, \\
P_{2}= & -2 \mu^{2} z^{2}+2 \mu(\lambda-2) z-\lambda^{2}+4 \lambda+2 \mu^{2}-4, \\
P_{3}= & 6 \mu^{3} z^{3}+6 \mu^{2}(-\lambda+3) z^{2}+3 \mu\left(\lambda^{2}-7 \lambda-3 \mu^{2}+12\right) z- \\
& -\lambda^{3}+10 \lambda^{2}+7 \lambda \mu^{2}-33 \lambda-21 \mu^{2}+36 .
\end{aligned}
$$

ПРЕДЛОЖЕНИЕ 1. Полиномиальное решение $P(z)=P_{n}(z)$ уравнения (10) при $\mu \neq 0$ является решением уравнения (4) при одном из двух возможных значениนั $\epsilon= \pm 1$. 
ДокАЗАтЕльство. Прямое вычисление позволяет убедиться, что если $P(z)$ - peшение уравнения (10), то функция $\widetilde{P}(z)=z^{n}\left(P^{\prime}\left(z^{-1}\right)-\mu P\left(z^{-1}\right)\right)$ также является решением этого уравнения. При этом, если $P(z)$ - полином степени $n$, то $\widetilde{P}(z)-$ полином той же степени. Далее, найдя вронскиан уравнения (10), получим, что для любых двух решений $P$ и $\widetilde{P}$ этого уравнения имеет место соотношение

$$
z^{n} e^{-\mu\left(z+z^{-1}\right)}\left(P^{\prime}(z) \widetilde{P}(z)-\widetilde{P}^{\prime}(z) P(z)\right)=c=\text { const }
$$

где $c=0$ тогда и только тогда, когда $P$ и $\widetilde{P}$ линейно зависимы. Но если $\widetilde{P}(z)$ и $P(z)-$ полиномы, то левая часть выражения (25) будет константой только в том случае, когда эта константа равна нулю и, следовательно, $P^{\prime}(z) \widetilde{P}(z)=\widetilde{P}^{\prime}(z) P(z)$. Таким образом, $\widetilde{P}(z)=\alpha P(z)$ для некоторой константы $\alpha$. Ясно, что $\alpha \neq 0$, поскольку в противном случае $P^{\prime}(z)-\mu P(z)=0$, что при $\mu \neq 0$ не согласуется с уравнением (10). Следовательно, для линейного отображения $P(z) \rightarrow \widetilde{P}(z)$ полином $P_{n}(z)$ является собственным вектором с ненулевым собственным значением $\alpha$. Квадрат данного отображения легко вычисляется. Он представляет собой линейный дифференциальный оператор второго порядка, для которого полином $P_{n}(z)$ является собственным вектором с собственным значением $\alpha^{2}$, а это, как оказывается, согласуется с уравнением (10) в том и только в том случае, когда $4 \omega^{2} \alpha^{2}=1$. Поэтому $\widetilde{P}= \pm(2 \omega)^{-1} P$ для одного из выборов знака, что эквивалентно одному из уравнений (4) при $\epsilon= \pm 1$. Предложение доказано.

СлеДСТвИЕ 2. Полиномы $P_{n}(z)$, удовлетворяющие уравнению (10) при $\mu \neq 0$, не имеют корней на единичной окружности и в нуле.

ДокАзАтЕльство. Первое утверждение следствия вытекает из предложения 1 и леммы 2. Если же предположить, что $P_{n}(0)=0$, т. е. $a_{0}=0$, то, как следствие уравнения (18), получим $a_{1}=0$. Затем из уравнений (19) последовательно выводится, что $a_{k}=0$ для всех остальных $k$, так что $P_{n} \equiv 0$.

Для полиномов (24) знаковые константы $\epsilon$, выраженные через параметр $\mu$ и корень $\lambda$ спектрального уравнения $\Delta_{n}=0$, имеют вид

$$
\begin{array}{ll}
n=1: & \epsilon=\epsilon_{1}=2 \omega \lambda, \\
n=2: & \epsilon=\epsilon_{2}=\omega \mu^{-1}\left(-\lambda^{2}+2 \lambda+2 \mu^{2}\right), \\
n=3: & \epsilon=\epsilon_{3}=3^{-1} \omega \mu^{-2}\left(\lambda^{3}-7 \lambda^{2}-7 \lambda \mu^{2}+12 \lambda+12 \mu^{2}\right) .
\end{array}
$$

Несмотря на возрастающую с ростом $n$ длину таких формул, мы имеем $\epsilon_{n}^{2}=1$ как следствие спектральных уравнений и соотношения (23).

Теперь, используя формулу (6) и вышеприведенные явные представления для $\Delta_{n}$ и $P_{n}$, можно построить решения уравнения (1), порождаемые полиномами $(24)$. Эти решения задаются выражениями

$$
\Phi_{j}=-i \epsilon_{j} e^{-i \omega t} \frac{T_{j}}{\bar{T}_{j}},
$$

где

$$
T_{1}=(\lambda-\mu-1) \cos \frac{\omega t}{2}-i(\lambda+\mu-1) \sin \frac{\omega t}{2},
$$




$$
\begin{aligned}
T_{2}= & \lambda((\lambda-2) \cos \omega t-2 \mu)-i\left(-\lambda^{2}+2 \lambda+4 \mu^{2}\right) \sin \omega t, \\
T_{3}= & \left(-\lambda^{3}+10 \lambda^{2}+7 \lambda \mu^{2}-33 \lambda+6 \mu^{3}-21 \mu^{2}+36\right) \cos \frac{3 \omega t}{2}+ \\
& +3 \mu\left(\lambda^{2}-2 \lambda \mu-7 \lambda-3 \mu^{2}+6 \mu+12\right) \cos \frac{\omega t}{2}+ \\
& +i\left(\lambda^{3}-10 \lambda^{2}-7 \lambda \mu^{2}+33 \lambda+6 \mu^{3}+21 \mu^{2}-36\right) \sin \frac{3 \omega t}{2}+ \\
& +3 i \mu\left(-\lambda^{2}-2 \lambda \mu+7 \lambda+3 \mu^{2}+6 \mu-12\right) \sin \frac{\omega t}{2} .
\end{aligned}
$$

Для краткости мы будем называть полиномиальными также решения уравнения (1), полученные с помощью формулы (6), где $P$ есть полином от $z$.

ПрЕДЛОЖЕНИЕ 2. Все корни полинома $P_{n}$, удовлетворяющего уравнению (10), nростые.

Так как корни ненулевые, данное утверждение следует из единственности решения задачи Коши для уравнения (10) по аналогии с доказательством следствия 1.

СлеДСтвиЕ 3. Если $P_{n}(z) \not \equiv 0$ u $P_{n}(z)=0$ для некоторого $z$, mо $P_{n}\left(z^{-1}\right) \neq 0$.

Видно, что для полиномиальных решений выполнение уравнения (4) влечет за собой выполнение уравнения (10), а из него следует справедливость одного из двух возможных уравнений (4) при $\epsilon= \pm 1$. Но уравнение (10), опять-таки для полиномов, эквивалентно системе уравнений (18)-(20), которая разрешима только при наличии вещественного корня $\lambda$ у спектрального уравнения $\Delta_{n}(\lambda, \mu)=0$. С другой стороны, для полинома $P_{n}$ уравнение (4) само по себе эквивалентно некоторой системе линейных однородных уравнений для коэффициентов $a_{k}$. Ее можно записать в виде

$$
\begin{aligned}
& \epsilon(2 \omega)^{-1} a_{0}+\mu a_{n}=0, \\
& \epsilon(2 \omega)^{-1} a_{k}-(n-k+1) a_{n-k+1}+\mu a_{n-k}=0, \quad k=1,2, \ldots, n .
\end{aligned}
$$

Здесь матрица коэффициентов размера $(n+1) \times(n+1)$ имеет вид $\mathbb{G}_{n}+\epsilon(2 \omega)^{-1} \mathbb{I}$, где $\mathbb{G}_{n}=\mathbb{G}_{n}(\mu)=\left(g_{k, l}\right)$,

$$
\begin{aligned}
& g_{k, l}= \begin{cases}0, & \text { если } l<n+2-k, \\
\mu, & \text { если } l=n+2-k, \quad k=1, \ldots, n+1,\end{cases} \\
& g_{k+1, n+2-k}=k-n-1 \text { для } k=1, \ldots, n \text {. }
\end{aligned}
$$

Условие $\operatorname{det}\left(\mathbb{G}_{n}+\epsilon(2 \omega)^{-1} \mathbb{I}\right)=0$ разрешимости системы уравнений $(27)$, (28) снова представляет собой алгебраическое уравнение степени $n+1$, где на этот раз роль неизвестного (собственного значения матрицы $\mathbb{G}_{n}$ ) играет величина $\epsilon(2 \omega)^{-1}$, рассматриваемая как функция от $\mu$.

Связь между этим и предыдущим условиями разрешимости уравнения (9) в полиномах становится очевидной при учете следующего матричного тождества:

$$
\mathbb{G}_{n}^{2} \equiv \mu^{2} \mathbb{I}-\mathbb{H}_{n}
$$


по модулю соотношения (23) (из чего, помимо прочего, следует представление $\mathbb{H}_{n}$ в виде произведения двух коммутирующих матричных сомножителей $\left.\pm \mathbb{G}_{n}+\mu \mathbb{I}\right)$. Повидимому, проще всего в справедливости (29) можно убедиться с помощью покомпонентного представления фигурирующих в нем матриц через символы Кронекера, а именно воспользовавшись формулами

$$
\begin{aligned}
\left(\mathbb{H}_{n}\right)_{j k} & =(n-j) \mu \delta_{j, k-1}-j(n+1-j) \delta_{j, k}+j \mu \delta_{j, k+1}, \quad j, k=0,1, \ldots, n . \\
\left(\mathbb{G}_{n}\right)_{j k} & =\mu \delta_{j, n-k}-j \delta_{j, n+1-k},
\end{aligned}
$$

Из тождества (29) вытекает факторизация

$$
\mathbb{H}_{n}+\left((2 \omega)^{-2}-\mu^{2}\right) \mathbb{I}=-\left(\mathbb{G}_{n}+(2 \omega)^{-1} \mathbb{I}\right)\left(\mathbb{G}_{n}-(2 \omega)^{-1} \mathbb{I}\right)
$$

следовательно, $\operatorname{det}\left(\mathbb{H}_{n}+\lambda \mathbb{I}\right)=(-1)^{n+1} \operatorname{det}\left(\mathbb{G}_{n}+(2 \omega)^{-1} \mathbb{I}\right) \operatorname{det}\left(\mathbb{G}_{n}-(2 \omega)^{-1} \mathbb{I}\right)$. Положим $\Delta_{n}^{( \pm)}=\operatorname{det}\left(\mathbb{G}_{n} \pm(2 \omega)^{-1} \mathbb{I}\right)$. При условии выполнения уравнения $(23)$

$$
\Delta_{n}(\lambda, \mu)=(-1)^{n+1} \Delta_{n}^{(+)}\left((2 \omega)^{-1}, \mu\right) \cdot \Delta_{n}^{(-)}\left((2 \omega)^{-1}, \mu\right)
$$

ЗАмЕчАниЕ 3. Из уравнения (27) и предложения 1 вытекает, что $a_{n} \neq 0$, т. е. степень полиномов $P_{n}$ равна $n$.

Для первых значений индекса $n$ имеем

$$
\begin{aligned}
& \Delta_{1}^{( \pm)}=(2 \omega)^{-2} \mp(2 \omega)^{-1}-\mu^{2}, \\
& \Delta_{2}^{( \pm)}= \pm(2 \omega)^{-3}+\mu(2 \omega)^{-2} \mp\left(\mu^{2}+2\right)(2 \omega)^{-1}-\mu^{3}, \\
& \Delta_{3}^{( \pm)}=(2 \omega)^{-4} \mp 2(2 \omega)^{-3}-\left(2 \mu^{2}+3\right)(2 \omega)^{-2} \pm 2\left(\mu^{2}+3\right)(2 \omega)^{-1}+\mu^{4} .
\end{aligned}
$$

Совпадение парных произведений выписанных выше полиномов с полиномами (22) в соответствии с уравнением (30) может быть проверено прямым вычислением.

Существование вещественных величин $\lambda$, которые обеспечивают совместность уравнений (18)-(20), т. е. вещественных собственных значений матриц $\mathbb{H}_{n}$, следует из трехдиагональности этих матриц и положительности произведения всех пар взаимно симметричных относительно диагонали внедиагональных элементов (на самом деле здесь положительны уже сами сомножители). Такие матрицы называются якобиевыми. С помощью операции сопряжения, использующей некоторую диагональ- 
ную матрицу, любую якобиеву матрицу можно преобразовать с сохранением набора собственных чисел в симметричную матрицу (см. [21], раздел 1.3.10), а у последней все собственные числа вещественны.

Кроме того, для симметричной матрицы число различных собственных векторов (в нашем случае это линейно преобразованные конечные последовательности $\left.a_{0}, \ldots, a_{n}\right)$ равно ее размерности. Уравнения $(18),(19)$ позволяют по известному значению $\lambda$ определить соответствующие $a_{0}, \ldots, a_{n}$ однозначно с точностью до общего множителя. И, поскольку все эти последовательности различны (они образуют базис), все собственные значения $\lambda$ также различны. Кроме того, преобразование сопряжения с матрицей $\operatorname{diag}(1,-1,1,-1, \ldots)$ изменяет знак недиагональных элементов на противоположный, сохраняя диагональ, что в нашем случае эквивалентно смене знака у параметра $\mu$. При этом детерминант также не меняется. Таким образом, получаем следующий результат.

ТеОрема 1. Имеют место следующие утверждения.

1. Полином $\Delta_{n}(\lambda, \mu)$ содержит только четные степени параметра $\mu$.

2. Для любого вещественного значения $\mu \neq 0$ все корни $\lambda$ спектральных уравнений $\Delta_{n}(\lambda, \mu)=0$ вещественные и простые.

ПреДЛОЖЕНИЕ 3. Для всех $n \in \mathbb{N}$ и при $\mu \neq 0$ мы имеем $\Delta_{n}\left(-\mu^{2}, \mu\right) \neq 0$, m.e. у спектрального уравнения нет корней $\lambda$, для которых уравнение (23) было бы неразрешимо относительно $\omega$.

ДокАЗАТЕЛЬСтво. При $\lambda=-\mu^{2}$ одно из решений уравнения (10) имеет вид $P=e^{\mu z}$ (это немедленно следует из уравнения (13) при учете "определения" $\lambda$ в (10), но легко проверяется и прямой подстановкой). Другое решение имеет вид $P=e^{\mu z} \int z^{n} e^{\mu\left(-z+z^{-1}\right)} d z$. Ни первое, ни второе решение, ни любая их линейная комбинация полиномами не являются. В то же время, если $\lambda$ - решение спектрального уравнения, то полиномиальное решение уравнения (10) должно существовать. Следовательно, $\lambda=-\mu^{2}$ решением спектрального уравнения не является.

СЛЕДСТВИЕ 4. Для любого заданного значения $\mu>0$ и целого $n>0$ два уравнения $\Delta_{n}^{( \pm)}\left((2 \omega)^{-1}, \mu\right)=0$ обладают совместно максимально возможсным количеством $2(n+1)$ различных вещественных корней $(2 \omega)^{-1}$, половина из которых положительны, а остальные получаются из них обращением знака.

Каждый из этих $n+1$ положительных корней порождает полиномиальное решение уравнения (10). Следствие 4 означает, что каждое вещественное собственное значение матрицы $\mathbb{H}$ соответствует вещественному собственному значению матрицы $\mathbb{G}$. Данная связь является следствием предложения 2, по существу утверждающего, что $\mathbb{H}$ и $\mathbb{G}$ имеют общую систему собственных векторов. Вещественный собственный вектор матрицы $\mathbb{H}$ является собственным вектором вещественной матрицы $\mathbb{G}$, и соответствующее ему собственное значение вещественно.

Некоторую информацию о расположении корней спектрального уравнения можно получить с помощью так называемых теорем Гершгорина [22]. Первая из них утверждает, что корни характеристического уравнения для матрицы с элемента- 
ми $h_{j k}$ лежат в кругах $D_{j}$ на комплексной плоскости с центрами ${ }^{1)} c_{j}=-h_{j j}$ и радиусами $r_{j}=\sum_{k \neq j}\left|h_{j k}\right|$ (круги Гершгорина). Для матрицы $\mathbb{H}_{n}$ имеем $c_{0}=0$ и $c_{j}=j(n+1-j)>0$ для $j=1,2, \ldots, n$, радиус $r_{0}=|\mu|$, а остальные радиусы $r_{1}, \ldots, r_{n}$ одинаковы и равны $|\mu|(n+2)$. Все точки $c_{j}$, кроме $c_{0}$, расположены на положительной вещественной полуоси и, поскольку $c_{j}=c_{n+1-j}$, группируются в несовпадающие пары одинаковых точек (являющихся центрами двух идентичных кругов $\left.D_{j}=D_{n+1-j}\right)$. Исключение составляет отдельно расположенный центр $c_{0}=0$, а также существующий только при нечетном $n$ тоже однократный центр $c_{(n+1) / 2}=(n+1)^{2} / 4$.

Установленная выше вещественность корней, вытекающее из уравнения (23) условие $\lambda>-\mu^{2}$ и элементарные геометрические соображения приводят к следующему результату.

ПРЕДЛОЖЕНИЕ 4. Все корни $\lambda$ спектрального уравнения лежат на вещественной оси внутри отрезка с левой границей $-\min \left(|\mu|, \mu^{2}\right)$ и правой границей, при четном $n$ равной $(n+2) / 4-|\mu|(n+2)$, а при нечетном $n$ равной $(n+1)^{2} / 4-|\mu|(n+2)$.

Расстояние $d_{j}$ между соседними центрами $c_{j}$ и $c_{j+1}$ кругов Гершгорина равно $|n-2 j|, j=0,1, \ldots, n-1$. Последовательность $\left\{d_{j}\right\}$ начинается с $n$, монотонно убывает до $d_{(n+1) / 2}=1$ для нечетного $n$ или до $d_{n / 2-1}=2$ для четного $n$, а затем также монотонно возрастает, повторяя в обратном порядке (геометрически для тех же пар точек) пройденные значения, до $d_{n-1}=n-2$ включительно. При четном $n$ формально нулевое расстояние $d_{n / 2}=0$ соответствует паре совпадающих кругов, которые в данном случае оказываются соседними в смысле нумерации. Точки $c_{0}$ и $c_{n}$, хотя и разнесены по индексам, являются геометрически соседними, расстояние между ними равно $n$, при этом $c_{n}$ совпадает с $c_{1}$, образуя пару совпадающих центров двух идентичных кругов. Сумма радиусов отдельного круга и соседней пары совпадающих кругов или двух соседних пар совпадающих кругов равна $|\mu|(n+3)$, когда один из кругов есть $D_{0}$, и равна $2|\mu|(n+2)$ в остальных случаях. Таким образом, если $r_{0}+r_{1}=|\mu|(n+3)<d_{0}=n$, то $D_{0}$ не пересекается с $D_{1}$, а также и с остальными кругами. Если к тому же $\rho_{j} \equiv r_{j}+r_{j+1}=2|\mu|(n+2)<\min d_{j}=1$ для нечетного $n$ или $\rho_{j}<\min d_{j}=2$ для четного $n$, то все круги Гершгорина либо не пересекаются, либо совпадают, причем совпадать с любым кругом может не более чем еще один.

Теорема 2. При $|\mu|<n /(n+3)$ спектральное уравнение имеет в интервале $\left(-\mu^{2},|\mu|\right)$ ровно один вещественный корень. Если $|\mu|<(n+2)^{-1} / 2$, то остальнье корни парами принадлежат отрезкам вещественной оси с центрами $j(n+1-j)$ и длиной $2|\mu|(n+2)$, за исключением, при нечетном $n$, дополнительного корня на отрезке с иентром $(n+1)^{2} / 4$ и той же длиной.

ДокАзАТЕЛЬСтво основывается на второй теореме Гершгорина, которая утверждает, что число корней внутри связного объединения нескольких кругов Гершгорина, не пересекающегося с не включенными в него кругами, равно числу входящих в данное объединение кругов [22]. При выполнении первого условия теоремы такой уединенной областью является круг с центром в нуле и радиусом $|\mu|$

1) При нашем определении величины $\lambda$ она является собственным значением, как оно понимается в [22], взятым с обратным знаком (см. формулу (21)). Поэтому координаты центров кругов имеют обратный знак по сравнению со значениями, приводимыми в [22]. 
(левая граница на самом деле есть $-\mu^{2}$ ), и в нем содержится ровно один корень. При выполнении второго условия теоремы это же справедливо для круга с центром $c_{(n+1) / 2}=(n+1)^{2} / 4$ и радиусом $|\mu|(n+2)<1 / 2$. Остальные корни парами лежат в непересекающихся парах совпадающих кругов.

Таким образом, мы показали, что у уравнения (1) существуют решения, выражающиеся через рациональные функции от $z=e^{i \omega t}$, т. е. через отношения тригонометрических полиномов, в случае, когда $n=-1-B / \omega$ есть целое неотрицательное число, а вещественные параметры $\mu=A / 2 \omega, \omega>0$, удовлетворяют одному из алгебраических уравнений $\Delta_{n}^{( \pm)}\left((2 \omega)^{-1}, \mu\right)=0$.

В рамках использовавшейся выше постановки задачи данный результат заведомо неполон, поскольку условия возникновения полиномиальных решений резко различаются для положительных и отрицательных $n$ (т. е. по существу для положительных и отрицательных $B$ ). А именно, если $n$ отрицательно, то уравнение (17) полиномиальных решений иметь не может. Между тем "с точки зрения уравнения (1)" положительные и отрицательные $B$ в сущности играют идентичные роли. Действительно, если $\phi(t)$ есть решение уравнения (1), то $-\phi(t+\pi / \omega)$ есть решение аналогичного уравнения, где $B$ заменено на $-B$. Если $\phi(t)$ представимо через тригонометрические полиномы при целом положительном $n$, то $-\phi(t+\pi / \omega)$ также представимо аналогичным образом, хотя в этом случае $n$ - отрицательное (но тоже целое) число.

Данное несоответствие является кажущимся и устраняется вследствие того, что существует преобразование

$$
P_{n}(z) \rightarrow \widetilde{P}(z)=z^{-n} e^{\mu\left(z+z^{-1}\right)}\left(P_{n}^{\prime}(-z)-\mu P_{n}(-z)\right),
$$

отображающее решения уравнения (17) в решения такого же уравнения, в котором $n$ заменено на $-n-2$. Если $n$ положительно, то $-n-2$ отрицательно, и $\widetilde{P}(z)$, хоть и представляет собой решение уравнения, аналогичного (17), как и следовало ожидать, полиномом не является за счет множителя перед скобками. Тем не менее соответствующим образом модифицированное уравнение (4) справедливо. А именно, как легко проверить, $\widetilde{P}^{\prime}(z)-\mu \widetilde{P}(z)=(-1)^{n}(2 \omega)^{-1} \epsilon z^{-n-2} \widetilde{P}\left(z^{-1}\right)$. Это есть уравнение вида (4), где $n$ заменено на $-n-2$ и $\epsilon$ заменено на $(-1)^{n} \epsilon$. Соответственно вместо соотношения (6) мы имеем $\widetilde{\Phi}=(-1)^{n+1} i \epsilon z^{n+1} \widetilde{P}(z) / \widetilde{P}\left(z^{-1}\right)$, что определяет решение уравнения (1), в котором $B$ заменено на $-B$. Это решение также может быть построено из тригонометрических полиномов.

ЗАмЕчАниЕ 4. Весьма неожиданно оказывается, что производная порядка $n+1$ любого решения $P(z)$ уравнения (10) является решением того же уравнения, что и (31), а именно, уравнения (10), в котором $n$ заменено на $-n-2$. Действительно, дифференцируя (10) $k \geqslant 1$ раз, получим

$$
\begin{aligned}
0= & z^{2} \frac{d^{k+2}}{z^{k+2}} P+\left((2 k-n) z+\mu\left(1-z^{2}\right)\right) \frac{d^{k+1}}{d z^{k+1}} P+ \\
& +(k(k-1-n)+\lambda+\mu(n-2 k) z) \frac{d^{k}}{d z^{k}} P+\mu k(n-k+1) \frac{d^{k-1}}{d z^{k-1}} P,
\end{aligned}
$$

и наше утверждение получается, если здесь подставить $k=n+1$ (при других $k$ уравнение существенно отличается от (10)). Для полиномиального решения данная 
зависимость бессодержательна, для второго решения, линейно от него не зависящего, она описывает связь с решениями родственного (отличающегося значением одного параметра) уравнения.

Из приведенных выше результатов вытекает

Теорема 3. Положим $M_{\mathrm{P}}^{(n)}=\left\{(\mu, \omega) \in \mathbb{R}^{2}: \operatorname{det}(\mathbb{H}+\lambda \mathbb{I})=0\right\}, n=0,1, \ldots$, где $4 \omega^{2}\left(\lambda+\mu^{2}\right)=1$ и $\mu \neq 0$. Рассмотрим два вложения $j_{ \pm}: M_{\mathrm{P}}^{(n)} \rightarrow \mathbb{R}^{3}$ такие, что

$$
(\mu, \omega) \rightarrow(A, B, \omega), \quad \text { де } \quad A=2 \omega \mu, \quad B=\mp(n+1) \omega .
$$

Тогда каждой точке множества $M_{\mathrm{P}}=\bigcup_{n} j_{ \pm} M_{\mathrm{P}}^{(n)}$ отвечает решение уравнения (1), для которого $e^{i \phi(t)}$ есть отношение тригонометрических полиномов от $\omega t$ для четного $n$ и от $\omega t / 2$ для нечетного $n$.

СлеДСтвиЕ 5. Решения уравнения (1) указанного выше вида существуют при $B=\tilde{n} \omega$ для всех ненулевых целых $\tilde{n}$ и произвольно выбираемого значения отношения $A / \omega \neq 0$.

Для полиномиальных решений $n$-го порядка уравнения (1) функция $e^{i \phi(t)}$ является периодической по переменной $t$ с периодом $T=2 \pi / \omega$ (эта функция совпадает с правой частью равенства (6), в котором $P=P_{n}, z=e^{i \omega t}$ ), и асимптотическая скорость изменения $\phi(t)$ в единицах $2 \pi$ для любого $t$ просто совпадает с $(2 \pi T)^{-1}(\phi(t+T)-\phi(t))$. Поэтому число вращения $N_{n}$ для таких решений равно $(2 \pi)^{-1} \int_{0}^{T} d \phi(t)$. Из равенства (6) получаем

$$
i d \phi=-(n+1) \frac{d z}{z}+\frac{P_{n}^{\prime}(z)}{P_{n}(z)} d z-\frac{P_{n}^{\prime}\left(z^{-1}\right)}{P_{n}\left(z^{-1}\right)} d z^{-1} .
$$

Когда $t$ пробегает интервал длины $T$, переменная $z=e^{i \omega t}$ совершает оборот по единичной окружности в направлении против часовой стрелки, а переменная $z^{-1}$ такой же оборот в противоположном направлении. Поэтому вклад в вариацию $\phi$ от первого слагаемого в $(32)$ равен $-2 \pi(n+1)$, а от второго и третьего сводится к "нормализованным" контурным интегралам, значения которых различаются знаком, давая при вычитании одинаковые вклады. Следовательно,

$$
N_{n}=-(n+1)+\frac{2}{2 i \pi} \oint_{|z|=1} \frac{d P_{n}(z)}{P_{n}(z)} .
$$

Все корни полинома $P_{n}$ не лежат на единичной окружности. Пусть $\nu_{-}$корней находятся внутри единичной окружности и $\nu_{+}-$снаружи, $\nu_{+}+\nu_{-}=n$. Приведенная выше формула утверждает, что $N_{n}=-(n+1)+2 \nu_{-}$. Тем самым справедлива

ТЕОрема 4. Для полиномиалъных решений уравнения (1) порядка $n$ при $\mu \neq 0$ число вращения является иелым: оно равно $\nu_{-}-\nu_{+}-1$.

СлЕДСТвиЕ 6. Для всех натуральных $n$ числа вращения удовлетворяют условиям

$$
\frac{N_{2 n}+1}{2} \in\{-n,-n+1, \ldots, n\}, \quad \frac{N_{2 n-1}}{2} \in\{-n,-n+1, \ldots, n-1\} .
$$


В приложении В показано, что числа вращения $N_{n}$ принимают все $[(n+1) / 2]+1$ значений начиная с $-(n+1)$ с шагом 2 .

Рассмотрим теперь задачу о резонансном захвате применительно к полиномиальным решениям уравнения (1). Конструктивный критерий, который можно использовать, когда известно хотя бы одно решение уравнения (1) на интервале изменения переменной $t$ длиной в период, предложен в работе [23] (см. также статью [16]). Он сводится к условию $|D| \gtrless 1$, где

$$
\begin{gathered}
D=-\frac{1}{2}(\operatorname{Im} F(T) \operatorname{Im} F(0))^{-1 / 2} \operatorname{Im}\left[e^{-i \delta \phi / 2}(F(T)-\overline{F(0)})\right], \\
\delta \phi=\phi(T)-\phi(0), \quad F(t)=\mathcal{I}_{Q}(t)+i e^{-\mathcal{I}_{P}(t)}, \\
\mathcal{I}_{P}(t)=\int \cos \phi(t) d t,
\end{gathered}
$$

и $T$ - период функции $q(t)$ в системе $(3)(T=2 \pi / \omega$ для уравнения $(1))$.

В нашем случае мы воспользуемся по существу эквивалентным, но более наглядным "прямым" методом. Мы покажем, что полиномиальные решения уравнения (1) принадлежат границе зоны захвата в пространстве параметров. Но для этого мы должны в достаточной мере охарактеризовать и другие решения уравнения (1), существующие для данных значений параметров, помимо полиномиального.

Свойства резонансного захвата для решений уравнения (1) можно описать с помощью связанного с ним отображения Пуанкаре, т. е. отображения

$$
S^{1} \rightarrow S^{1}: e^{i \phi(0)} \rightarrow e^{i \phi(T)}
$$

где $\phi(t)$ пробегает множество решений для всех возможных начальных данных $\phi(0)$. Для вычисления этого отображения мы воспользуемся формулой (16), где функция $P(z)$, описывающая произвольное (общее) решение уравнения $(10)$, представляется в соответствии с уравнением (25) через его полиномиальное решение:

$$
P(z)=P_{n}(z)\left(a+b \mathcal{I}_{n}(z)\right)
$$

где

$$
\mathcal{I}_{n}(z)=\int_{1}^{z} w^{n} e^{\mu\left(w+w^{-1}\right)} P_{n}(w)^{-2} d w, \quad-\pi<\arg z<\pi,
$$

и, следовательно $\mathcal{I}_{n}(1)=0$. Здесь $a, b$ суть произвольные вещественные числа, по крайней мере одно из которых не равно нулю. В (34) интегрирование может производиться, вообще говоря, по произвольной кривой, соединяющей точки 1 и $z$, хотя она не должна проходить через корни полинома $P_{n}$ или в них оканчиваться (в последнем случае, когда $P_{n}(z)=0$, выражение $\mathcal{I}_{n}$ не определено ${ }^{2)}$ ). Для полной определенности будем считать, что путь интегрирования от 1 до $z$ составлен из сегмента дуги единичной окружности длиной меньше $\pi$ и отрезка прямой радиального направления, оканчивающегося в точке $z$. Такой путь определен значением $z$, не лежащим на отрицательной вещественной полуоси, однозначно. Нам здесь достаточно считать, что $z$ лежит настолько близко к единичной окружности, чтобы данный путь интегрирования не встречал корней полинома $P_{n}$, которые, как было

${ }^{2)}$ В интересующем нас случае $\mathcal{I}_{n}(z)$ имеет в корнях полинома $P_{n}$ простые полюсы. 
показано ранее, все находятся вне нее. Определенная таким образом аналитическая функция $P(z)$ является вещественной.

Подставив теперь (33) в соотношение (16) и воспользовавшись уравнениями вида (4) и $(9)$, которым полином $P_{n}(z)$ удовлетворяет, получим

$$
\Phi_{(a, b)}(z)=z^{-n-1} \frac{P_{n}(z)}{P_{n}\left(z^{-1}\right)} \frac{Q_{(a, b)}^{(+)}(z)}{Q_{(a, b)}^{(-)}(z)},
$$

где

$$
Q_{(a, b)}^{( \pm)}(z)=(\epsilon \mp i) a+2 \omega e^{\mu\left(z+z^{-1}\right)}\left(P_{n}(z) P_{n}\left(z^{-1}\right)\right)^{-1} b+\left(\epsilon \mathcal{I}_{n}\left(z^{\mp 1}\right) \mp i \mathcal{I}_{n}\left(z^{ \pm 1}\right)\right) b .
$$

Функция (35) также удовлетворяет уравнению (14), принимает унимодулярные значения на единичной окружности и потому определяет в соответствии с соотношением $e^{i \phi_{(a, b)}(t)}=\Phi_{(a, b)}\left(e^{i \omega t}\right)$ вещественную функцию $\phi_{(a, b)}(t)$, удовлетворяющую уравнению (1) до тех пор, пока равенство (35) определяет голоморфную функцию (фактически остается непрерывным на дуге единичной окружности, см. ниже). Выберем теперь произвольное значение $\phi_{0} \in[0,2 \pi)$. Легко убедиться, что если положить

$$
a=-P_{n}(1)^{-2} \sin \frac{\phi_{0}}{2}, \quad b=(2 \omega)^{-1} e^{-2 \mu}\left(\cos \frac{\phi_{0}}{2}+\epsilon \sin \frac{\phi_{0}}{2}\right),
$$

то $\Phi_{(a, b)}(1)=e^{i \phi_{0}}$.

Таким образом, равенство (35) описывает, по крайней мере при достаточно малых значениях $t$, решение задачи Коши для уравнения (1) при произвольном начальном условии. Соответственно, для описания отображения Пуанкаре $S^{1} \rightarrow S^{1}$ остается найти значение функции (35), которое достигается при подходе по кривой $z=e^{i \omega t}$ к значению аргумента $t=T$ снизу при том дополнительном условии, что на кривой при промежуточных значениях $t$ эта функция непрерывна.

Поясним смысл последней фразы. Дело в том, что имеется существенное различие между общим решением вида (35) уравнения (14) и полиномиальным (частным) решением, описывающимся аналогичным уравнением (6) с $P(z)=P_{n}(z)$. Это различие заключается в том, что выражение в правой части формулы (35) не является аналитической функцией на единичной окружности и, следовательно, в ее окрестности при $b \neq 0$ и ненулевом (вещественном при вещественном $P_{n}$ ) значении интеграла

$$
\mathcal{I}_{n}^{(0)}=i^{-1} \oint_{|w|=1} w^{n} e^{\mu\left(w+w^{-1}\right)} \frac{d w}{P_{n}(w)^{2}} .
$$

Областью определения данной аналитической функции является риманова поверхность с точками ветвления в нуле и в бесконечности. При проецировании на комплексную плоскость в значении, которое принимает функция (35), возникает неоднозначность, и при обходе окружности при возвращении в исходную точку будет наблюдаться конечный разрыв, связанный с неоднозначностью значений интеграла (34) (в силу его зависимости от расположения пути интегрирования по отношению к обходимому сингулярному центру $z=0$ ). При этом все остальные компоненты выражений в правых частях равенств (35) и (36) суть однозначные и непрерывные в окрестности единичной окружности функции. 
При описанном выше выборе пути интегрирования разрыв будет происходить при пересечении точкой $z$ отрицательной вещественной полуоси, когда направление, в котором проходится при интегрировании дуга окружности, меняется на противоположное, и сама эта дуга заменяется на свое зеркальное отражение относительно горизонтальной оси. Таким образом, представление (35), (36) непосредственно применимо лишь при $t<T / 2$.

Однако формулу (35) можно сделать пригодной для определения $\phi(t)$ в соответствии с равенством (15) и на интервале $T / 2<t<T$, т. е. в итоге на всем интервале $0 \leqslant t<T$, если при таких значениях $t$ интерпретировать в соотношении (36) $\mathcal{I}\left(z^{ \pm 1}\right)=\mathcal{I}\left(e^{i \omega t}\right)$ не как функцию из $(33)$, а как ее аналитическое продолжение через разрез вдоль отрицательной вещественной полуоси. Такие продолжения описываются выражениями $\mathcal{I}_{n}(z)+i \mathcal{I}_{n}^{(0)}$ для $\mathcal{I}_{n}(z)$ и $\mathcal{I}_{n}\left(z^{-1}\right)-i \mathcal{I}_{n}^{(0)}$ для $\mathcal{I}_{n}\left(z^{-1}\right)$. Теперь легко получить требуемое значение:

$$
e^{i \phi_{(a, b)}(T)}=\left.\Phi_{(a, b)}\left(e^{i \omega t}\right)\right|_{t \rightarrow T-0}=e^{i \phi_{0}} \frac{1+(2 \omega)^{-1} e^{-2 \mu}\left(e^{-i \phi_{0}}-i \epsilon\right) P_{n}(1)^{2} \mathcal{I}_{n}^{(0)}}{1+(2 \omega)^{-1} e^{-2 \mu}\left(e^{i \phi_{0}}+i \epsilon\right) P_{n}(1)^{2} \mathcal{I}_{n}^{(0)}}
$$

Это та точка на окружности, в которую попадает точка $e^{i \phi_{0}}$ в результате ее перемещения вдоль интегральной кривой векторного поля, задаваемого уравнением (1), при приращении переменной $t$, равном одному периоду $T$. Иными словами, мы получили явную формулу отображения Пуанкаре для уравнения (1) в случае существования полиномиального решения.

Теорема 5. Отображение Пуанкаре для уравнения (1) в случае существования полиномиалъного решения обладает одной неподвижной точкой при $\mathcal{I}_{n}^{(0)} \neq 0$ и является тождественным отображением при $\mathcal{I}_{n}^{(0)}=0$.

ДокАЗАТЕЛЬСТВА требует лишь единственность неподвижной точки. Согласно формуле (38) точка $e^{i \phi_{0}}$ отображается в себя, если и только если дробь в правой части равенства (38) равна единице. Для этого (равные) вещественные части комплексно-сопряженных числителя и знаменателя должны быть отличны от нуля (иначе дробь равна -1), а мнимые части (различающиеся знаком), наоборот, должны обращаться в нуль. Таким образом, при $\mathcal{I}_{n}^{(0)} \neq 0$ необходимо выполнение равенства $\sin \phi_{0}=-\epsilon$. Но, так как $\epsilon^{2}=1$, это означает, что $\cos \phi_{0}=0$, и поэтому $e^{i \phi_{0}}=-i \epsilon$. Дробь в этом случае действительно равна единице. Таким образом, значение $e^{i \phi_{0}}$ с данным свойством, характеризующим неподвижную точку отображения Пуанкаре существует. Из самого вычисления следует, что оно единственно. Это то, что и требовалось доказать.

ЗАмечаниЕ 5. Хотя в формулировке теоремы 5 используется значение $\mathcal{I}_{n}^{(0)}$, величиной, не зависящей от нормировки полинома $P_{n}$, является $P_{n}(1)^{2} \mathcal{I}_{n}^{(0)}$. Эту величину мы будем называть инвариантом отображения Пуанкаре. Именно она входит в формулу (38) и, следовательно, определяет отображение Пуанкаре.

Известно, что отображения Пуанкаре для уравнения типа (1), в том числе, когда в его правой части стоит функция $f(t)$ более общего вида, принадлежат группе Мёбиуса $P S U(1,1)$ изометрий плоскости Лобачевского, сохраняющих ориентацию. В модели Пуанкаре плоскости Лобачевского автоморфизмы, индуцируемые данными преобразованиями на граничной окружности, реализуют соответствующие преобразования Пуанкаре. Этот фундаментальный результат был получен в работе [7] 
с помощью анализа группы голономий связности Эресмана в главном (тривиальном) расслоении, моделирующем фазовое пространство неголономной системы, которая соответствует уравнению, обобщающему (1). Элементарное доказательство этого результата содержится в статье [23]. Преобразования группы Мёбиуса могут допускать две (гиперболический случай), одну (параболический случай) или ни одной (эллиптический случай) неподвижной точки, а также быть тождественным отображением. Здесь неподвижная точка соответствует решению уравнения (1), периодическому с периодом $T$ с точностью до приращения, цельнократного $2 \pi$; при тождественном отображении Пуанкаре все решения обладают таким свойством. При этом гиперболический и эллиптический случаи соответствуют наличию резонансного захвата и псевдохаотическому поведению ${ }^{3)}$ соответственно. Мы видели выше, что для полиномиальных решений число вращения является целым. Тем же свойством обладают и решения уравнения (1), описывающие резонансный захват [12], [13], в то время как для псевдохаотического случая число вращения нецелое. Но для решений первого типа реализуется параболический случай отображения Пуанкаре, а для вторых - гиперболический. Следовательно, полиномиальные решения, ввиду непрерывной зависимости числа вращения от параметров, дают внешние предельные точки для области, в которой реализуется резонансный захват.

Что касается значения контурного интеграла $\mathcal{I}_{n}^{(0)}$, собственно и определяющего отображение Пуанкаре для полиномиального решения, то для его вычисления мы можем использовать тот факт, что в интегрируемом выражении знаменатель является полиномом. Воспользуемся разложением на простые дроби

$$
P_{n}(z)^{-1}=\sum_{k=1}^{n} \frac{1}{P_{n}^{\prime}\left(z_{k}\right)} \cdot \frac{1}{z-z_{k}},
$$

справедливым для любого полинома $P_{n}$, имеющего только простые корни $z_{k}$, что как раз имеет место в нашем случае. Возводя это равенство в квадрат и выражая $P_{n}^{\prime}\left(z_{k}\right)$ через $P_{k}\left(z^{-1}\right) \neq 0$ в соответствии с уравнением типа $(4)$, получим

$$
P_{n}(z)^{-2}=\sum_{k=1}^{n} \frac{\mathcal{P}_{k}^{(2)}}{\left(z-z_{k}\right)^{2}}+2 \sum_{k=1}^{n} \frac{\mathcal{P}_{k}^{(1)}}{z-z_{k}}
$$

где

$$
\mathcal{P}_{k}^{(2)}=(2 \omega)^{2} \frac{z_{k}^{-2 n}}{P_{n}\left(z_{k}^{-1}\right)^{2}}, \quad \mathcal{P}_{k}^{(1)}=(2 \omega)^{2} \frac{z_{k}^{-n}}{P_{n}\left(z_{k}^{-1}\right)} \sum_{\substack{1 \leqslant l \leqslant n, l \neq k}} \frac{z_{l}^{-n}}{\left(z_{k}-z_{l}\right) P_{n}\left(z_{l}^{-1}\right)}
$$

Таким образом, подынтегральное выражение в (34) (и в (37)) может быть представлено в виде

$$
w^{n} e^{\mu\left(w+w^{-1}\right)} P_{n}(w)^{-2} d w=-d L+\sum_{k=1}^{n} L_{k} d w
$$

\footnotetext{
3) При псевдохаотическом поведении имеет место "скрытый" периодический процесс, однако отображение Пуанкаре в общем случае “считывает" его состояние через промежутки времени, несоизмеримые с периодом процесса. Поэтому как отображение Пуанкаре, так и график функции $\phi(t)$, не обнаруживают каких-либо видимых признаков периодичности и выглядят вполне беспорядочными. Такая картина резко контрастирует с гиперболическим случаем, когда имеются два $T$ периодических, после вычитания линейной функции, решения, а все остальные экспоненциально сходятся к одному из них (второе играет ту же роль при “обращении времени").
} 
где

$$
\begin{aligned}
L & =w^{n} e^{\mu\left(w+w^{-1}\right)} \sum_{k=1}^{n} \frac{\mathcal{P}_{k}^{(2)}}{w-z_{k}} \\
L_{k} & =\frac{e^{\mu\left(w+w^{-1}\right)}}{w-z_{k}}\left[\mathcal{P}_{k}^{(2)}\left(n w^{n-1}-\mu w^{n-2}\right)+\left(\mu \mathcal{P}_{k}^{(2)}+2 \mathcal{P}_{k}^{(1)}\right) w^{n}\right] .
\end{aligned}
$$

Преобразуя далее $L_{k}$, воспользуемся тем фактом, что, как известно, экспонента $e^{a\left(x \pm x^{-1}\right) / 2}$ является производящей функцией для функций Бесселя с целыми индексами. В частности, имеет место разложение $e^{\mu\left(z+z^{-1}\right)}=\sum_{m=-\infty}^{\infty} z^{m} I_{m}(2 \mu)$, где $I_{m}(\cdot)$ - модифицированные функции Бесселя первого рода, и ряд сходится при любом $z \neq 0$ (см. справочник [24], п. 9.6.33). При неограниченном увеличении индекса $m$ в сторону как положительных, так и отрицательных значений “коэффициенты" $I_{m}(2 \mu)=I_{-m}(2 \mu)$ (для целого $\left.m\right)$ сходятся к нулю, причем достаточно быстро для того, чтобы ряд, получающийся при подстановке данного разложение в (37), можно было интегрировать почленно. Подставляя его, получим

$$
L_{k}=\sum_{m=-\infty}^{\infty} Y_{k ; m} \frac{z^{m}}{w-z_{k}}
$$

где

$$
Y_{k ; m}=\mathcal{P}_{k}^{(2)}\left(n I_{m-n+1}(2 \mu)-\mu I_{m-n+2}(2 \mu)\right)+\left(\mu \mathcal{P}_{k}^{(2)}+2 \mathcal{P}_{k}^{(1)}\right) I_{m-n}(2 \mu) .
$$

Имеют место тождества

$$
\begin{aligned}
\frac{z^{m}}{z-a}=\frac{a^{m}}{z-a}+\sum_{j=0}^{m-1} a^{m-j-1} z^{j} \quad \text { при } m \geqslant 0, \\
\frac{z^{m}}{z-a}=\frac{a^{m}}{z-a}-\sum_{j=m}^{-1} a^{m-j-1} z^{j} \quad \text { при } m \leqslant-1 .
\end{aligned}
$$

В результате их применения к разложению (41) возникают два вида рядов. Первые слагаемые в (42) порождают ряд вида $\sum_{m=-\infty}^{\infty} x^{m} I_{m}(a)$, сворачивающийся в упоминавшуюся "производящую экспоненту" $e^{\mu\left(x \pm x^{-1}\right) / 2}$. Остальные вклады порождают аналогичные ряды с "однонаправленным" изменением индекса $0 \leqslant m<\infty$, которые мы оформим как функции

$$
\Sigma_{l}(z, \mu)=\sum_{m=0}^{\infty} z^{m} I_{m+l}(2 \mu), \quad \Sigma_{l}(z, a, \mu)=\sum_{j=0}^{\infty} \sum_{m=0}^{\infty} z^{j} a^{m} I_{j+m+l}(2 \mu),
$$

где $l \in \mathbb{N}$ и $z, a \in \mathbb{C}, \mu \in \mathbb{R}$. Это целые функции аргумента $z$, и нам здесь понадобится лишь это их свойство ${ }^{4)}$. Применяя (42), (43), для (41) можно получить следующее представление:

$$
\begin{aligned}
L_{k} \equiv & \mathcal{P}_{k}^{(3)} z_{k}^{n} e^{\mu\left(z_{k}+z_{k}^{-1}\right)}\left(w-z_{k}\right)^{-1}- \\
& -\mathcal{P}_{k}^{(2)}\left(n z_{k}^{-1}-\mu z_{k}^{-2}\right) \Sigma_{1-n}(w, \mu)+\mathcal{P}_{k}^{(2)} \mu z_{k}^{-1} \Sigma_{2-n}(w, \mu)+\mathcal{P}_{k}^{(3)} \Sigma_{1-n}\left(w, z_{k}, \mu\right)- \\
& -w^{-1} z_{k}^{-1}\left(\mathcal{P}_{k}^{(2)}\left(n-\mu z_{k}^{-1}\right) \Sigma_{n}\left(w^{-1}, \mu\right)-\right. \\
& \left.\quad-\mathcal{P}_{k}^{(2)} \mu \Sigma_{n-1}\left(w^{-1}, \mu\right)+\mathcal{P}_{k}^{(3)} \Sigma_{n+1}\left(w^{-1}, z_{k}^{-1}, \mu\right)\right),
\end{aligned}
$$

\footnotetext{
4) Формула 5.8.3.1 из каталога [25] позволяет получить для них интегральные представления.
} 
где

$$
\mathcal{P}_{k}^{(3)}=\left(\mu+n z_{k}^{-1}-\mu z_{k}^{-2}\right) \mathcal{P}_{k}^{(2)}+2 \mathcal{P}_{k}^{(1)}
$$

Сопоставление разложений (39), (44) и формул (33), (34) приводит к одному важному для рассматриваемого вычисления следствию. А именно, как следствие (39) и (44), в правой части (33) стоит произведение $P_{n}(z)=\mathrm{const} \cdot \prod_{k=1}^{n}\left(z-z_{k}\right)$ и суммы (умноженных на константу) функций $L$, заданных в (40), и $n$ интегралов от функций $L_{k}$ (см. (44)). Результат может быть сингулярным в нуле (за счет вклада от третьей и четвертой строк в (44)), в бесконечности (за счет вклада второй строки), но также и в каждой точке $z=z_{k}$ за счет вклада от первой строки. А именно, в результате интегрирования в каждой из этих точек, вообще говоря, присутствует ветвление благодаря выражению вида $\left(z-z_{k}\right) \ln \left(z-z_{k}\right)$, умноженному на аналитическую в окрестности $z_{k}$ функцию. Однако в рассматриваемом нами случае, когда соотношение (33) описывает решение уравнения (10), сингулярности в корнях $P_{n}(z)$, в противоположность сингулярностям в нуле и бесконечности, возникать не могут, в этих точках все решения аналитичны. Назревающее противоречие не реализуется лишь в одном случае, суть которого выражает следующий факт.

ПреДЛОЖЕНИЕ 5. Если полином $P_{n}(z)$ удовлетворяет уравнению (10), то его корни ограничены алгебраическими связями $\mathcal{P}_{k}^{(3)}=0, k=1,2, \ldots, n$, которые могут быть записаны в двух эквивалентных развернутых формах:

$$
\begin{aligned}
& \left(\mu+n z_{k}^{-1}-\mu z_{k}^{-2}\right) P_{n}^{\prime}\left(z_{k}\right)^{-1}+2 \sum_{\substack{1 \leqslant l \leqslant n, l \neq k}}\left(z_{l}-z_{k}\right)^{-1} P_{n}^{\prime}\left(z_{l}\right)^{-1}=0, \\
& \left(\mu+n z_{k}^{-1}-\mu z_{k}^{-2}\right) z_{k}^{-n} P_{n}\left(z_{k}^{-1}\right)^{-1}+2 \sum_{\substack{1 \leqslant l \leqslant n, l \neq k}}\left(z_{l}-z_{k}\right)^{-1} z_{l}^{-n} P_{n}\left(z_{l}^{-1}\right)^{-1}=0 .
\end{aligned}
$$

Замечательным образом условие обращения в нуль функции $\mathcal{P}_{k}^{(3)}$, определенной в (45), призванное по существу лишь устранить первую строку в (44), заодно обращает в нуль оба выражения в (44), в которые входит функция $\Sigma$ с тремя аргументами. Теперь при вычислении контурного интеграла в (37) остаток второй строки, являясь целой функцией, вклада в результат не дает, а третья строка дает легко вычисляемые (например, после подстановки определений (43) функций $\Sigma$ через ряды) вклады, пропорциональные $\Sigma_{n}(0, \mu)=I_{n}(2 \mu)$ и $\Sigma_{n-1}(0, \mu)=I_{n-1}(2 \mu)$. Итогом является следующий результат.

Теорема 6. Справедливо следующее соотношение:

$$
\mathcal{I}_{n}^{(0)}=8 \pi \mu \omega^{2}\left(I_{n+1}(2 \mu) \sum_{k=1}^{n} z_{k}^{-2 n-1} P_{n}\left(z_{k}^{-1}\right)^{-2}+I_{n}(2 \mu) \sum_{k=1}^{n} z_{k}^{-2 n-2} P_{n}\left(z_{k}^{-1}\right)^{-2}\right)
$$

Выборочные численные оценки при малых $n$ не обнаруживают нетривиальных нулей функции $\mathcal{I}_{n}^{(0)}$. 


\section{Демонстрационные примеры}

\section{ПРИЛОЖЕНИЕ А}

A.1. Случай $n=1$. В уравнении $\Delta_{1}=0$ (см. формулы (22)) удобно рассматривать $\omega$ как изначально задаваемый параметр. С учетом (26) получаем $\lambda=\epsilon(2 \omega)^{-1}$. Из уравнений (10), (24) вытекает, что $\mu=(2 \omega)^{-1} \sqrt{1-2 \epsilon \omega}$, поэтому $A=\sqrt{1-2 \epsilon \omega}$ и $B=-2 \omega$. Допустимо также значение $\mu$, имеющее обратный знак по сравнению с приведенным, но мы здесь будем рассматривать преобразование $\mu \rightarrow-\mu$ как тривиальное. Полином

$$
P_{1}=(2 \omega)^{-1}(\sqrt{1-2 \epsilon \omega} z+2 \omega-\epsilon)
$$

имеет единственный корень $z_{1}=\epsilon \sqrt{1-2 \epsilon \omega}$. Решение уравнения (1) определяется из соотношения

$$
e^{i \phi}=-i \epsilon \frac{(1-\epsilon \sqrt{1-2 \omega \epsilon}) \cos (\omega t / 2)+i(1+\epsilon \sqrt{1-2 \omega \epsilon}) \sin (\omega t / 2)}{(1-\epsilon \sqrt{1-2 \omega \epsilon}) \cos (\omega t / 2)-i(1+\epsilon \sqrt{1-2 \omega \epsilon}) \sin (\omega t / 2)} e^{-i \omega t} .
$$

Оно корректно определено, если либо $\epsilon=1$ и $0<\omega<1 / 2$, либо $\epsilon=-1$ и $\omega>0$. В первом случае $\left|z_{1}\right|<1$, во втором $\left|z_{1}\right|>1$. Соответственно в первом случае число вращения $N=0$, во втором $N=-2$. Инвариант отображения Пуанкаре равен

$$
P_{1}(1)^{2} \mathcal{I}_{1}^{(0)}=2 \pi \omega^{-1}(1-\epsilon \omega \sqrt{1-2 \epsilon \omega})(1-2 \epsilon \omega)^{-1 / 2}\left(\epsilon \sqrt{1-2 \epsilon \omega} I_{n+1}(2 \mu)+I_{n}(2 \mu)\right) .
$$

A.2. Случай $n=2$. Здесь проще всего в качестве произвольного параметра взять $\lambda$. Спектральное уравнение дает $\omega^{2}=(\lambda-1) \lambda^{-3}$. Следует различать две возможности: $\lambda>1$ и $\lambda<0$.

А.2.1. Случай $\lambda>1$. В этом случае

$$
\begin{gathered}
\omega=\lambda^{-1} \sqrt{1-\lambda^{-1}}, \quad \mu=-\frac{\epsilon}{2}(\lambda-2)\left(1-\lambda^{-1}\right)^{-1 / 2}, \\
A=\epsilon\left(-1+2 \lambda^{-1}\right), \quad B=-3 \lambda^{-1} \sqrt{1-\lambda^{-1}}, \\
P_{2}=-\frac{1}{2}(\lambda-2)^{2}(\lambda-1)^{-1}\left(\lambda z^{2}+2 \epsilon \lambda \sqrt{1-\lambda^{-1}} z+\lambda-2\right) .
\end{gathered}
$$

Корни полинома $P_{2}$ равны $z_{1,2}=\lambda^{-1 / 2}(-\epsilon \sqrt{\lambda-1} \pm 1)$, причем, поскольку $\left|z_{1,2}\right|^{2}-1=$ $\mp 2 \epsilon \lambda^{-1} \sqrt{\lambda-1}$, один из них находится внутри единичной окружности, а другой снаружи. Решения уравнения (1) определяются из уравнения

$$
\begin{aligned}
e^{i \phi}= & -i \epsilon \frac{\epsilon \sqrt{\lambda} \sqrt{\lambda-1}+(\lambda-1) \cos \left(\lambda^{-1} \sqrt{1-\lambda^{-1}} t\right)+i \sin \left(\lambda^{-1} \sqrt{1-\lambda^{-1}} t\right)}{\epsilon \sqrt{\lambda} \sqrt{\lambda-1}+(\lambda-1) \cos \left(\lambda^{-1} \sqrt{1-\lambda^{-1}} t\right)-i \sin \left(\lambda^{-1} \sqrt{1-\lambda^{-1}} t\right)} \times \\
& \times e^{-i \lambda^{-1} \sqrt{1-\lambda^{-1}} t} .
\end{aligned}
$$

Для них число вращения $N=-1$. Инвариант отображения Пуанкаре равен

$$
P_{2}(1)^{2} \mathcal{I}_{2}^{(0)}=-2 \pi \sqrt{\lambda-1}(\epsilon \sqrt{\lambda}+\sqrt{\lambda-1})^{2}\left(-\sqrt{\lambda-1} I_{3}(2 \mu)+\epsilon \lambda^{3 / 2}(\lambda-2)^{-1} I_{2}(2 \mu)\right) .
$$


А.2.2. Случай $\lambda<0$. По аналогии с предыдущим случаем, положив $-\lambda=\tilde{\lambda}>0$, имеем

$$
\begin{gathered}
\omega=\tilde{\lambda}^{-1} \sqrt{1+\tilde{\lambda}^{-1}}, \quad \mu=-\frac{\epsilon}{2}(2+\tilde{\lambda})\left(1+\tilde{\lambda}^{-1}\right)^{-1 / 2}, \\
A=-\epsilon\left(1+2 \tilde{\lambda}^{-1}\right), \quad B=-3 \tilde{\lambda}^{-1} \sqrt{1+\tilde{\lambda}^{-1}}, \\
P_{2}=-\frac{1}{2}(2+\tilde{\lambda})^{2}(1+\tilde{\lambda})^{-1}\left(\tilde{\lambda} z^{2}-2 \epsilon \tilde{\lambda} \sqrt{1+\tilde{\lambda}^{-1}} z+\tilde{\lambda}+2\right) .
\end{gathered}
$$

Корни полинома $P_{2}$ равны $z_{1,2}=\tilde{\lambda}^{-1 / 2}(\epsilon \sqrt{1+\tilde{\lambda}} \pm i)$, и, поскольку $\left|z_{1}\right|^{2}=\left|z_{2}\right|^{2}=$ $z_{1} z_{2}=1+2 \tilde{\lambda}^{-1}$, оба корня находятся вне единичной окружности. Решение уравнения (1) определяется из соотношения

$$
\begin{aligned}
e^{i \phi}= & -i \epsilon \frac{\epsilon \sqrt{\tilde{\lambda}} \sqrt{1+\tilde{\lambda}}+(1+\tilde{\lambda}) \cos \left(\tilde{\lambda}^{-1} \sqrt{1+\tilde{\lambda}^{-1}} t\right)+i \sin \left(\tilde{\lambda}^{-1} \sqrt{1+\tilde{\lambda}^{-1}} t\right)}{\epsilon \sqrt{\tilde{\lambda}} \sqrt{1+\tilde{\lambda}}+(1+\tilde{\lambda}) \cos \left(\tilde{\lambda}^{-1} \sqrt{1+\tilde{\lambda}^{-1}} t\right)-i \sin \left(\tilde{\lambda}^{-1} \sqrt{1+\tilde{\lambda}^{-1}} t\right)} \times \\
& \times e^{-i \tilde{\lambda}^{-1} \sqrt{1+\tilde{\lambda}^{-1}} t} .
\end{aligned}
$$

Число вращения $N=-3$. Инвариант отображения Пуанкаре равен

$$
P_{2}(1)^{2} \mathcal{I}_{2}^{(0)}=2 \pi \sqrt{1+\tilde{\lambda}}(-\epsilon \sqrt{\tilde{\lambda}}+\sqrt{1+\tilde{\lambda}})^{2}\left(\sqrt{1+\tilde{\lambda}} I_{3}(2 \mu)+\epsilon \tilde{\lambda}^{3 / 2}(\tilde{\lambda}+2)^{-1} I_{2}(2 \mu)\right) .
$$

Таким образом, в случае $n=2$ при данном $\omega$ мы получили три решения уравнения (1), одно из которых имеет число вращения $N_{2}=-3$, а для двух других $N_{2}=-1$. Следовательно, уже при $n=2$ не реализуется возможное значение $N_{2}=1$ (см. следствие 6).

\section{Характеристики полиномиальных решений}

ПРИЛОЖКНИЕ Б

Пусть $\phi(t)=\phi(t ; A, B, \omega)$ - решение уравнения (1) и

$$
\langle\cos \phi\rangle=\lim _{k \rightarrow \infty} T^{-1} \int_{k T}^{(k+1) T} \cos \phi(t) d t, \quad\langle\sin \phi\rangle=\lim _{k \rightarrow \infty} T^{-1} \int_{k T}^{(k+1) T} \sin \phi(t) d t .
$$

Если говорить об интерпретации этих усреднений, легко видеть [12], что если точка $(A, B, \omega)$ принадлежит области резонансного захвата, то $\langle\sin \phi\rangle=B-\omega N$, где $N$ есть число вращения $N(A, B, \omega)$; это же справедливо и для граничных точек, которым соответствуют полиномиальные решения. Из второго уравнения системы (3) следует, что

$$
\langle\cos \phi\rangle=\lim _{k \rightarrow \infty} T^{-1}(\xi((k+1) T)-\xi(k T)) .
$$

Из формул (4), (6) для $z=e^{i \omega t}$ и, следовательно, $d t=(i \omega z)^{-1} d z$ вытекает, что

$$
\frac{1}{2}\left(e^{i \phi} \pm e^{-i \phi}\right) d t= \pm P\left(z^{-1}\right)^{-1} d P\left(z^{-1}\right)+P(z)^{-1} d P(z)-\mu\left(d z \pm d z^{-1}\right) .
$$

Если $P(z)=P_{n}(z)$ есть полином, то функции $\cos \phi(t)$ и $\sin \phi(t)$, пропорциональные $\left(e^{i \phi} \pm e^{-i \phi}\right)$, являются периодическими с периодом $T$, и вместо предела можно 
взять любой элемент последовательности. Интегрируя левую часть равенства (48) по интервалу $(0, T)$, а правую - по единичной окружности, получим

$$
T^{-1} \int_{0}^{T} \frac{1}{2}\left(e^{i \phi} \pm e^{-i \phi}\right) d t=( \pm 1-1) i \omega \nu_{-},
$$

где $\nu_{-}$- число корней полинома $P_{n}$ внутри единичной окружности. Таким образом, для полиномиальных решений

$$
\langle\cos \phi\rangle=T^{-1} \int_{0}^{T} \cos \phi(t) d t=0, \quad\langle\sin \phi\rangle=T^{-1} \int_{0}^{T} \sin \phi(t) d t=-2 \omega \nu_{-} .
$$

Так как $|\langle\sin \phi\rangle|<1$ и $\nu_{-} \geqslant 0$, при $\omega>0$ имеем $\nu_{-}<(2 \omega)^{-1} ;$ соответственно, если $\omega>1 / 2$, то $\nu_{-}=0$ и, как следствие, $N_{n}=-n-1$.

\section{Предельный случай $A \rightarrow 0$}

ПРИЛОЖКНИЕ В

В случае $A=0$ и соответственно $\mu=0$, уравнение (10) при фиксированном натуральном $n$ обладает $n$ решениями вида $P(z)=P_{n}^{[k]}(z)=z^{k}, k=1, \ldots, n$, для которых $\lambda=\lambda_{k}=k(n+1-k)$. Это также "полиномиальные" решения, хотя, в отличие от случая $\mu \neq 0$, здесь степень полинома может быть не равна $n$. Соответствующие решения уравнения (1), получающиеся путем рутинного применения формулы (16), имеют вид

$$
\begin{aligned}
e^{i \phi} & =\frac{\sqrt{k} z^{-k+(n+1) / 2}-i \sqrt{n+1-k} z^{k-(n+1) / 2}}{\sqrt{k} z^{k-(n+1) / 2}+i \sqrt{n+1-k} z^{-k+(n+1) / 2}}= \\
& =z^{-2 k+n+1} \frac{1-i \sqrt{k^{-1}(n+1-k)} z^{2 k-n-1}}{1+i \sqrt{k^{-1}(n+1-k)} z^{-2 k+n+1}},
\end{aligned}
$$

где $z=e^{i \omega t}$, а $\omega=\omega_{k}$ определяется из уравнения $4 \omega_{k}^{2} \lambda_{k}=1$, т. е.

$$
\begin{aligned}
& \omega_{k}=\frac{(k(n+1-k))^{-1 / 2}}{2}, \\
& B= \begin{cases}-(n+1) \omega=-(n+1) \frac{(k(n+1-k))^{-1 / 2}}{2}<-1 & \text { при } k \neq \frac{n+1}{2}, \\
-1 & \text { при } k=\frac{n+1}{2},\end{cases}
\end{aligned}
$$

для нечетного $n$. Заметим, что из (49) напрямую вытекает явное представление ${ }^{5}$

$$
\phi(t)=(n+1-2 k) \omega t-2 \operatorname{arctg} \frac{\sqrt{n+1-k} \cos (2 k-(n+1)) \omega t}{\sqrt{k}+\sqrt{n+1-k} \sin (2 k-(n+1)) \omega t},
$$

однако при $k<n+1-k$ оно пригодно лишь на ограниченном интервале значений $t$.

5) Заметим также, что при $A=0$ общее решение уравнения (1) представимо в элементарных функциях. 
Представление (49) удобно для вычисления числа вращения. Действительно, в формуле (49) числитель представляет собой равномерно вращающийся вектор длины $r=\sqrt{k^{-1}(n+1-k)}$, приложенный к точке $(1,0)$. Поскольку $k\left(r^{2}-1\right)=n+1-2 k$, при $k<(n+1) / 2$ окружность, описываемая этим вектором, охватывает центр $(0,0)$ и не охватывает его при $k>(n+1) / 2$. Кроме того, имеется исключительный случай $k=(n+1) / 2$ при нечетном $n$; в этом случае кривая, описываемая вектором $e^{i \phi}$, вырождается в точку. Таким образом, при $k<(n+1) / 2$ каждый оборот вектора $z=e^{i \omega t}$ дает вклад величиной в один оборот во вращение вектора $e^{i \phi}$. Знаменатель действует аналогично и удваивает эффект. В итоге число вращения оказывается равным $(n+1-2 k)+2(2 k-n-1)=2 k-n-1$. При выборе различных допустимых значений $k$ оно изменяется с шагом 2 в пределах от $1-n$ до -1 для четного $n$ и до -2 для нечетного $n$. При $k \geqslant(n+1) / 2$ дробь не вносит суммарного вклада во вращение вектора $e^{i \phi}$, и число вращения равно $n+1-2 k$. Оно меняется от $1-n$ (при максимальном $k$ ) до -1 для четного $n$ и до 0 для нечетного $n$ (при минимальном $k$ ).

Мы видим, что интервал целых чисел с шагом 2 от $-n+1$ до -1 для четного $n$ и до -2 для нечетного $n$ покрывается дважды, и при нечетном $n$ имеется дополнительно однократное значение числа вращения, равное нулю. Сравнение показывает, что все соответствующие значения $\lambda$ фактически описываются теоремой 2 (где следует положить $j=k$ ) для случая исчезающе малого $\mu$. Однако в теореме 2 указано еще одно значение $\lambda$, а именно изолированный корень $\lambda \in\left(-\mu^{2}, \mu\right)$, который стремится к нулю при $\mu \rightarrow 0$. Ему не соответствует ни одно из решений $P_{n}^{[k]}(z)$. Но число вращения для него, в том числе при сколь угодно малых, хоть и ненулевых, $\mu$, существует, и его легко определить. Действительно, из уравнения (23) следует, что при уменьшении $\mu$ в рассматриваемом случае $\omega \rightarrow \infty$. В приложении Б было показано, что при $\omega>1 / 2$ число вращения равно $-n-1$. Это значение и реализуется для рассматриваемого $\lambda$, по крайней мере при достаточно малых $\mu$.

Подведем итог. Значения числа вращения при нулевом или достаточно малом $\mu$ заполняют с шагом 2 интервал целых чисел от $-n-1$ до 0 включительно. Покрытие двукратно (одному числу вращения соответствуют две функции $\phi(t)$ ) для всех значений числа вращения, кроме $-n-1$, а также, при нечетном $n$, нуля. Поскольку целое число вращения непрерывно зависит от параметра $\mu$, этот факт остается справедливым и для произвольных $\mu$. Одновременно мы получаем значения чисел $\nu_{ \pm}$ корней полинома $P_{n}(z)$ вне и внутри единичной окружности. Эти характеристики полиномиального решения определяются по тому, к какому из значений $\lambda_{k}$ (или к нулю) приближается соответствующий ему корень $\lambda$ спектрального уравнения при $\mu \rightarrow 0$ или, эквивалентно, каков его порядковый номер в упорядоченной по возрастанию последовательности корней.

Благодарности. Авторы выражают благодарность И. В. Нетаю и А. А. Гайфуллину за полезные обсуждения.

\section{Список литературы}

[1] В. М. Бухштабер, О. И. Карпов, С. И. Тертычный, УМН, 67:1(403) (2012), 181-182.

[2] W. C. Stewart, Appl. Phys. Lett., 12 (1968), 277-280.

[3] D. E. McCumber, J. Appl. Phys., 39 (1968), 3113-3118.

[4] В. В. Шмидт, Введение в физику сверхпроводников, МЦНМО, М., 2000. 
[5] B. D. Josephson, Phys. Lett., 1:7 (1962), 251-253.

[6] А. Бароне, Дж. Патерно, Эффект Джсоефсона. Физика и применение, Мир, М., 1984.

[7] R. Foote, Rep. Math. Phys., 42:1-2 (1998), 249-271, arXiv: math/9808070.

[8] R. L. Foote, M. Levi, S. Tabachnikov, Tractrices, bicycle tire tracks, Hatchet planimeters, and a 100-year-old conjecture, arXiv: 1207.0834 .

[9] J. Guckenheimer, Yu. S. Ilyashenko, Mosc. Math. J., 1:1 (2001), 27-47.

[10] В.И. Арнольд, Дополнительные главы теории обыкновенных дифференииальных уравнений, Наука, М., 1978.

[11] В. И. Арнольд, Геометрические методы в теории обыкновенных дифференииальных уравнений, РХД, Ижевск; МЦНМО, М., 2002.

[12] В. М. Бухштабер, О. В. Карпов, С. И. Тертычный, ТМФ, 162:2 (2010), 254-265.

[13] Ю.С. Ильяшенко, Д.А. Рыжов, Д. А. Филимонов, Функи. анализ и его прил., 45:3 (2011), 41-54.

[14] Д. А. Рыжов, Резонансный захват и специальные эргодические теоремы, Дисс. ... канд. физ.-матем. наук, МГУ, М., 2012.

[15] В. М. Бухштабер, О. В. Карпов, С. И. Тертычный, УМН, 59:2(356) (2004), 187-188.

[16] S. I. Tertychniy, Long-term behavior of solutions of the equation $\dot{\phi}+\sin \phi=f$ with periodic $f$ and the modeling of dynamics of overdamped Josephson junctions: Unlectured notes, arXiv: math-ph/0512058.

[17] D. Schmidt, G. Wolf, "Double confluent Heun equation", Heun's differential equations. Part C, ed. A. Ronveaux, Oxford Univ. Press, Oxford, 1995.

[18] С. Ю. Славянов, В. Лай, Специальные функиии: единая теория, основанная на анализе особенностей, Невский диалект, СПб., 2002.

[19] S.I. Tertychniy, The modelling of a Josephson junction and Heun polynomials, arXiv: math-ph/0601064.

[20] S. I. Tertychniy, Electron. J. Diff. Equ., 2007:133 (2007), 1-20.

[21] В. П. Ильин, Ю. И. Кузнецов, Трехдиагональные матрищы и их приложения, Наука, M., 1985.

[22] Дж. Х. Уилкинсон, Алгебраическая проблема собственных значений, Наука, М., 1970.

[23] С. И. Тертычный, УМН, 55:1(331) (2000), 195-196.

[24] М. Абрамовиц, И. Стиган (ред.), Справочник по специалъным функииям с формулами, графиками и математическими таблицами, Наука, М., 1979.

[25] А.П. Прудников, Ю.А. Брычков, О.И. Маричев, Интеграль и ряды. Специалънъе функции, Наука, М., 1983. 